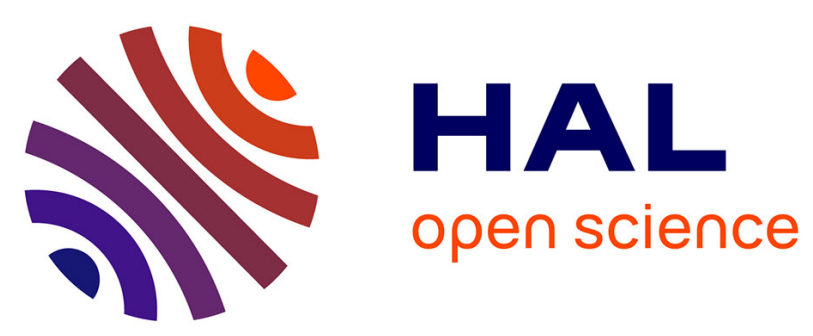

\title{
A robust algebraic approach to fault diagnosis of uncertain linear systems
}

Abdouramane Moussa Ali, Cédric Join, Frédéric Hamelin

\section{To cite this version:}

Abdouramane Moussa Ali, Cédric Join, Frédéric Hamelin. A robust algebraic approach to fault diagnosis of uncertain linear systems. 50th IEEE Conference on Decision and Control and European Control Conference, CDC-ECC 2011, Dec 2011, Orlando, Florida, United States. pp.CDROM. hal00650802

\section{HAL Id: hal-00650802 \\ https://hal.science/hal-00650802}

Submitted on 12 Dec 2011

HAL is a multi-disciplinary open access archive for the deposit and dissemination of scientific research documents, whether they are published or not. The documents may come from teaching and research institutions in France or abroad, or from public or private research centers.
L'archive ouverte pluridisciplinaire HAL, est destinée au dépôt et à la diffusion de documents scientifiques de niveau recherche, publiés ou non, émanant des établissements d'enseignement et de recherche français ou étrangers, des laboratoires publics ou privés. 


\title{
A robust algebraic approach to fault diagnosis of uncertain linear systems
}

\author{
Abdouramane Moussa Ali, Cédric Join and Frédéric Hamelin
}

\begin{abstract}
This article proposes an algebraic method to fault diagnosis for uncertain linear systems. The main advantage of this new approach is to realize fault diagnosis only from knowledge of input and output measurements without identifying explicitly model parameters. Using tools and results of algebraic identification and pseudospectra analysis, the issues of robustness of the proposed approach compared to the model order and noise measurement are examined. Numerical examples are provided and discussed to illustrate the efficiency of the proposed fault diagnosis method.
\end{abstract}

\section{INTRODUCTION}

Fault diagnosis methods include some actions implemented in order to detect, isolate and identify any abnormal phenomenon on a system.

In [3], [7] and references therein the classical approaches using analytical information are depicted. They allow robust fault diagnosis in the presence of unknown entries and parametric uncertainties. These methods depend not only on structural knowledge of the system, but also require knowledge of system parameters that can be more or less accurate.

The algebraic approach to fault diagnosis presented in [11] deals with actuator and sensor additive faults and requires only the knowledge of the system order. This article is devoted to analyse the robustness of this approach with respect to the model order and measurement noises.

Throughout the paper, we adopt a distributional formulation, as in [1]. It permits us to obtain explicit expressions in time domain, for the development of the approach.

The paper is organized as follows. In section 2, we fix some notations used in this paper. Different assumptions on the system structure and the fault signal structures are needed to solve the fault diagnosis problem. Section 3 is devoted to the outline of the approach discussed in this paper. In section 4 , the question of the robustness, with respect to system order, is addressed. Finally, the question of the robustness, with respect to measurement noise, is the object of section 5 before the conclusion.

\section{PRELIMINARIES AND PROBLEM FORMULATION}

In order to better understand the aim of this paper, let us begin with recall the outline of the proposed approach in

This work was not supported by any organization

A. Moussa Ali, C. Join and F. Hamelin are with Research Center for Automatic Control, Nancy-University, CNRS, 54506 Vandœuvre, France \{amoussaa, cjoin, fhamelin\}@cran.uhp-nancy.fr

A. Moussa Ali joined the project team signals and systems in physiology and engineering (sisyphe) of INRIA since June 2011 abdouramane.moussa_alieinria.fr

C. Join is also with the project team Non-asymptotic estimation for online systems of INRIA cedric.join@inria.fr
[11]. This approach is performed in a distributional framework using usual definitions and basic properties described in [12]. First, recall some definitions and results from the distribution theory and fix the notations we shall use in the sequel. Let $f$ be a locally measurable function on an open set of $\mathbb{R}$ denoted by $\mathcal{K}$. We define the regular distribution $T_{f}$, for all smooth functions $\phi$ with compact support in $\mathcal{K}$, by

$$
<T_{f}, \phi>=\int f(\tau) \phi(\tau) d \tau
$$

Derivation, delay and integration can be formed from the convolution product $y^{(1)}=\delta^{(1)} \star y, y(t-\tau)=\delta_{\tau} \star y$, $\int_{0}^{t} y(\tau) d \tau=H \star y$ and more generally

$$
\underbrace{\int_{0}^{t} \cdots \int}_{p \text { times }} y(\tau) d \tau^{p}=\underbrace{H \star \cdots \star H}_{p \text { times }} \star y=H^{\star p} \star y
$$

where $\delta$ is Dirac distribution, $\delta_{\tau}$ is Dirac distribution with delay $\tau$ and $H$ is the unit step function (Heaviside distribution). The distribution theory extends the concept of derivation to all integrable functions. If function $f$ is continuous except at point $x$ with a finite jump $s_{x}$, the associated distribution derivative is given by $\dot{T}_{f}=\dot{f}-s_{x} \delta_{x}$, where $\dot{f}$ is the usual derivative of function $f$ (defined over $\mathbb{R} \backslash\{x\})$. The next Theorem [12] is the main result from which the proposed fault diagnosis algorithm is developed.

Theorem 2.1: If a distribution $T$ has a compact support $\operatorname{Supp}(T)$ and a finite order $m$, the product $\phi T=0$ whenever the smooth function $\phi$ and all its derivatives of order $\leq m$ vanish on $\operatorname{Supp}(T)$.

According to this theorem, it follows $t^{k} \delta^{(n)}=0 \forall k>n$ because the support and order of Dirac $\delta^{n}$ are $\{0\}$ and $n$ respectively and $\frac{d^{n}}{d t^{n}}\left[t^{k}\right]_{t=0}=0 \forall k>n$. For $k \leq n$, we obtain

$$
t^{k} \delta^{(n)}=(-1)^{k} \frac{n !}{(n-k) !} \delta(n-k)
$$

The systems under consideration are those whose control signal $u_{r}(t)$ and output signal $y_{r}(t)$ satisfy a differential equation described by

$$
\left\{\begin{array}{l}
\dot{x}(t)=A x(t)+B u_{r}(t), x\left(t_{0}\right)=x_{0} \\
y_{r}(t)=C x(t)+D u_{r}(t)
\end{array}\right.
$$

where $x \in \mathbb{R}^{n}, u_{r} \in \mathbb{R}^{n_{u}}$ and $y_{r} \in \mathbb{R}^{n_{y}}$ are respectively the state vector, vector of real inputs (the actuator outputs) and the vector of real outputs provided by the system. System matrices $A, B, C, D$ and initial condition $x_{0}$ are unknown a priori. 
The system (2) can be brought, in distributional framework, into a set of MISO (multi inputs single output) models

$$
P \star y_{r_{j}}-\sum_{i=1}^{n_{u}} h_{j i} \star u_{r_{i}}=\phi_{j} \quad: \quad j=1, \cdots, n_{y}
$$

where $P$ and $h_{j i}$ are differential polynomial functions given by

$$
\begin{aligned}
P & =a_{n} \delta^{(n)}+a_{n-1} \delta^{(n-1)}+\cdots+a_{0} \delta \\
h_{j i} & =b_{n}^{j i} \delta^{(n)}+b_{n-1}^{j i} \delta^{(n-1)}+\cdots+b_{0}^{j i} \delta
\end{aligned}
$$

with scalars $a_{k}, b_{k}^{j i}$ related to the system parameters, $\phi_{j}$ a linear combination of Dirac distribution derivatives of order less or equal than $n-1$ containing the contribution of the initial conditions.

In the presence of actuator faults and sensor faults modeled respectively by causal functions $f_{a_{i}}\left(i=1, \cdots, n_{u}\right)$ and $f_{s_{j}}$ $\left(j=1, \cdots, n_{y}\right)$, then the control input $u(t)$ computed by the controller and the measured output $y(t)$ are given in terms of real variables and fault signals as follows :

$$
\begin{aligned}
& u_{r_{i}}=u_{i}+f_{a_{i}}, i=1, \cdots, n_{u} \\
& y_{r_{j}}=y_{j}-f_{s_{j}}, j=1, \cdots, n_{y}
\end{aligned}
$$

The faulty system is then modeled by

$$
P \star y_{j}-\sum_{i=1}^{n_{u}} h_{j i} \star u_{i}=P \star f_{s_{j}}+\sum_{i=1}^{n_{u}} h_{j i} \star f_{a_{i}}+\phi_{j}
$$

$j=1, \cdots, n_{y}$

We deal with fault signals modeled by structured functions [5]. The main fault signals found in literature (abrupt, ramp, intermittent faults) can be modeled as structured signals [7]. If faults $f_{a_{i}}$ and $f_{s_{j}}$ are structured, then there exists two differential polynomials $\Gamma_{a_{i}}$ and $\Gamma_{s_{j}}$ such that

$$
\Gamma_{a_{i}} f_{a_{i}}=\Gamma_{s_{j}} f_{s_{j}}=0
$$

For example the delayed Dirac $\delta_{\tau}$ and the delayed Heaviside step function $H(t-\tau)$ are structured and

$$
[t-\tau] \delta_{\tau}=\left[(t-\tau) \frac{d}{d t}\right] H(t-\tau)=0
$$

\section{FAULT DIAGNOSIS}

Consider the simple case where the fault to be detected is a bias on the actuator $\lambda$, then

$$
f_{a_{\lambda}}=l_{a_{\lambda}} H\left(t-\tau_{a_{\lambda}}\right)
$$

where $\tau_{a_{\lambda}}$ denoted the time occurrence and $l_{a_{\lambda}}$ the magnitude of the bias fault. We have

$$
h_{j, \lambda} \star f_{a_{\lambda}}=l_{a_{\lambda}}\left[b_{n} \delta_{\tau_{a_{\lambda}}}^{(n-1)}+\cdots+b_{1} \delta_{\tau_{a_{\lambda}}}+b_{0} H\left(t-\tau_{a_{\lambda}}\right)\right]
$$

where $b_{i}=b_{i}^{j, \lambda}, i=0, \cdots, n$.

According to theorem (2.1), we obtain the equalities

$$
\left[\left(t-\tau_{a_{\lambda}}\right)^{n+1} \frac{d}{d t}\right] h_{j, \lambda} \star f_{a_{\lambda}}=0 \text { and } t^{n} \phi_{j}=0
$$

The faulty system is given by (6) by setting $f_{s_{j}}=0$ and $f_{a_{i}}=0$ if $i \neq \lambda$ :

$$
P \star y_{j}-\sum_{i=1}^{n_{u}} h_{j, i} \star u_{i}=h_{j, \lambda} \star f_{a_{\lambda}}+\phi_{j} \quad: \quad j=1, \cdots, n_{y}
$$

To eliminate the singularities appearing in the faulty model, let us just multiply equation (12) by

$$
\Gamma=t^{n+1}\left(t-\tau_{a_{\lambda}}\right)^{n+1} \frac{d}{d t}
$$

which can be rewritten as follows

$$
\Gamma=\beta_{n+2} \Gamma_{n+2}-\beta_{n+1} \Gamma_{n+1}-\cdots-\beta_{1} \Gamma_{1}
$$

where

$$
\begin{aligned}
& \Gamma_{k}=t^{n+k} \frac{d}{d t} \\
& \beta_{k}=-\left(-\tau_{a_{\lambda}}\right)^{n+2-k} \frac{(n+1) !}{(k-1) !(n+2-k) !}
\end{aligned}
$$

$k=1, \cdots, n+2$

The multiplication of (12) by $\Gamma$ provides the equalities

$$
\Gamma\left[P \star y_{j}-\sum_{i=1}^{n_{u}} h_{j i} \star u_{i}\right]=0 \quad: \quad j=1, \cdots, n_{y}
$$

For $t \leq \tau_{a_{\lambda}}$, equality (16) is satisfied in spite of $\tau_{a_{\lambda}}\left(\tau_{a_{\lambda}}\right.$ is not identifiable before fault occurrence) because, in this case

$$
t^{n+1}\left[P \star y_{j}-\sum_{i=1}^{n_{u}} h_{j i} \star u_{i}\right]=0 \quad: \quad j=1, \cdots, n_{y}
$$

However, for $t>\tau_{a_{\lambda}}$, application of successive integrations allows to generate $m$ redundancy relations which can be rearranged to obtain the spectral equality on which the diagnosis task will be based

$$
\left[A_{j, n+2}-\beta_{n+1} A_{j, n+1}-\ldots-\beta_{1} A_{j, 1}\right] X=0
$$

where the line $\mu$ of matrix $A_{j, \nu} \in \mathbb{R}^{m \times(n+1)\left(n_{u}+1\right)}$ (completely defined according to measured signals $u$ and $y$ ) and vector $\mathrm{X}$ are respectively given by

$$
\left[\begin{array}{c}
H^{\star p+\mu} \star\left[\Gamma_{\nu} y_{j}^{(n)}\right] \\
\vdots \\
H^{\star p+\mu} \star\left[\Gamma_{\nu} y_{j}\right] \\
-H^{\star p+\mu} \star\left[\Gamma_{\nu} u_{n_{u}}^{(n)}\right] \\
\vdots \\
-H^{\star p+\mu} \star\left[\Gamma_{\nu} u_{n_{u}}\right] \\
\vdots \\
-H^{\star p+\mu} \star\left[\Gamma_{\nu} u_{1}^{(n)}\right] \\
\vdots \\
-H^{\star p+\mu} \star\left[\Gamma_{\nu} u_{1}\right]
\end{array}\right] \quad \text { and } \quad\left[\begin{array}{c}
a_{n} \\
\vdots \\
a_{0} \\
b_{n}^{j, n_{u}} \\
\vdots \\
b_{0}^{j, n_{u}} \\
\vdots \\
b_{n}^{j, 1} \\
\vdots \\
b_{0}^{j, n_{u}}
\end{array}\right]
$$

By taking $p \geq n+1$, integrations ensure the elimination of all derivatives (numerically less robust than integrations).

Then parameter $\beta_{i}(i=1, \ldots, n+1)$ can be estimated by computing the generalised eigenvalues of a couple of 
matrices $\left(B A_{j, n+2}, B A_{j, i}\right)$ where $B$ is a non-zero matrix verifying :

$$
B A_{j, n+2} \neq 0 \text { and } B A_{j, k}=0, \forall k \neq i
$$

The computation of this matrix $B$ can be achieved by $Q R$ factorization. To proceed like that, just take the number of redundancy relations $m>n(n+1)\left(n_{u}+1\right)$. The matrices couple $\left(B A_{j, n+2}, B A_{j, i}\right)$ has more than one generalised eigenvalue. When the fault occurs, one of these generalised eigenvalues becomes stationary. After estimating components of vector $\beta$, we may, according to them, estimate $\tau_{a_{\lambda}}$. For example, by means of (15b) we have

$$
\tau_{a_{\lambda}}=\frac{\beta_{n+1}}{n+1}
$$

which can be estimated also as generalised eigenvalue of the matrices couple $\left(B A_{j, n+2},(n+1) B A_{j, n+1}\right)$ where the nonzero matrix $B$ verified conditions (20) with $i=n+1$. Note $\theta$ the associated generalised eigenvector, normalized with $\theta_{\left(n_{u}-\lambda+1\right)(n+1)+1}=1$. Thanks to (1), (9) and (12) we obtain the equality

$$
\left[t^{n+1}\left(t-\tau_{a_{\lambda}}\right)^{n} \frac{d}{d t}\right]\left[P \star y_{j}-\sum_{i=1}^{n_{u}} h_{j i} \star u_{i}\right]= \pm b_{\lambda, n} l_{a_{\lambda}} \tau_{a_{\lambda}}^{n+1} n ! \delta_{\tau_{a_{\lambda}}}
$$

The estimation of $l_{a_{\lambda}}$ can be achieved, based on (22) and $\tau_{a_{\lambda}}$ estimation, by

$$
p ! \frac{\left[A_{j, n+1}(:, 1)+\sum_{k=0}^{n-1} C_{k}^{n}\left(-\tau_{a_{\lambda}}\right) A_{j, k+1}(:, 1)\right] \theta}{\tau_{a_{\lambda}}^{n+1} n !\left(t-\tau_{a_{\lambda}}\right)^{p}}
$$

In this equality as in the following, if it does not confusion, we use the same notations for exact values and estimates.

Like any diagnosis algorithm, the decision is based on the time evolution of estimates. Fault $f_{a_{\lambda}}$ is detected and isolated when estimates of $\tau_{a_{\lambda}}$ and $l_{a_{\lambda}}$ simultaneously become stationary. Let consider the case $n=2, n_{u}=1$ and $n_{y}=2$. The corresponding spectral equalities are

$$
\begin{aligned}
& {\left[A_{1,4}-3 \tau_{a_{\lambda}} A_{1,3}+3 \tau_{a_{\lambda}}^{2} A_{1,2}-\tau_{a_{\lambda}}^{3} A_{1,1}\right] X=0} \\
& {\left[A_{2,4}-3 \tau_{a_{\lambda}} A_{2,3}+3 \tau_{a_{\lambda}}^{2} A_{2,2}-\tau_{a_{\lambda}}^{3} A_{2,1}\right] X=0}
\end{aligned}
$$

rewritten as

$$
\begin{aligned}
& \left.\left[\begin{array}{ll}
A_{1,4} & -3 A_{1,3}
\end{array}\right]-\tau_{a_{\lambda}}^{2}\left[\begin{array}{ll}
-3 A_{1,2} & A_{1,1}
\end{array}\right]\right] \Theta=0 \\
& \left.\left[\begin{array}{ll}
A_{2,4} & -3 A_{2,3}
\end{array}\right]-\tau_{a_{\lambda}}^{2}\left[\begin{array}{ll}
-3 A_{2,2} & A_{2,1}
\end{array}\right]\right] \Theta=0
\end{aligned}
$$

where $\Theta=\left[\begin{array}{ll}X^{T} & \tau_{a_{\lambda}} X^{T}\end{array}\right]^{T}$.

$\tau_{a_{\lambda}}^{2}$ is estimated as a generalised eigenvalue of matrices couples

$$
\left(\left[\begin{array}{ll}
A_{1,4} & -3 A_{1,3}
\end{array}\right],\left[\begin{array}{ll}
-3 A_{1,2} & A_{1,1}
\end{array}\right]\right)
$$

and

$$
\left(\left[\begin{array}{ll}
A_{2,4} & -3 A_{2,3}
\end{array}\right],\left[\begin{array}{ll}
-3 A_{2,2} & A_{2,1}
\end{array}\right]\right)
$$

Figures (1) and (2) illustrate graphically the simulation results in noise-free case. The loop is closed according a $P I$ controller. The actuator fault occurs at time $\tau_{a_{1}}=1$ and is characterized by constant magnitude $l_{a_{1}}=-0.8$ on the actuator. When the estimated quantities are outliers, that is to say clearly far from the stationarity (e. g. fluctuations), we consider that fault has not occurred and set the values estimated at zero. As we can see on figures (2(a)) and (2(b)), independently of the output considered, the simulated fault is well detected since the estimates of $\tau_{a_{\lambda}}$ and $l_{a_{\lambda}}$ become stationary just after the fault occurrence, around the exact values.

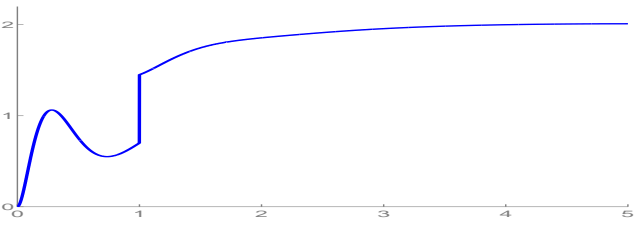

(a) Control input

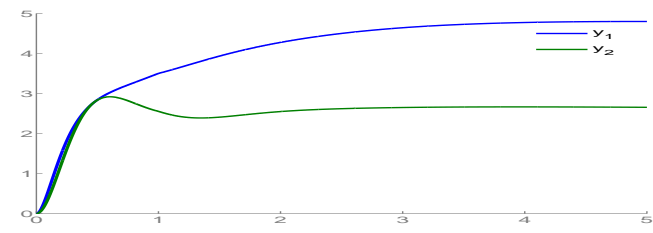

(b) Output

Fig. 1. System with two outputs and one input, in presence of an actuator bias

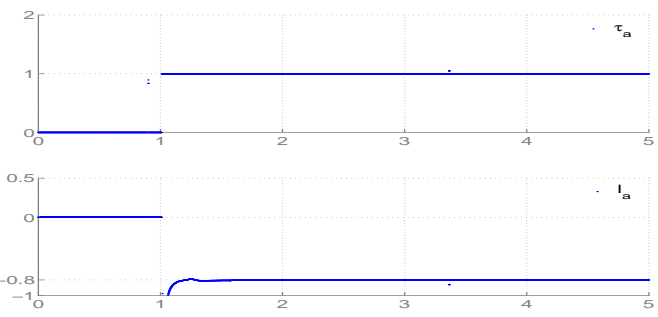

(a) $\tau_{a_{1}}$ (top) and $l_{a_{1}}$ (down) deduced from $y_{1}$

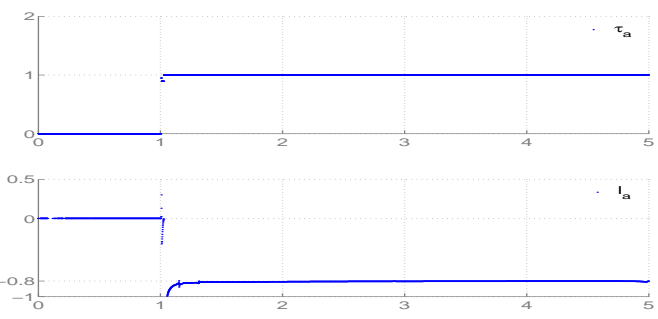

(b) $\tau_{a_{1}}$ (top) and $l_{a_{1}}$ (down) deduced from $y_{2}$

Fig. 2. Temporal evolution of estimates

Note that, based on temporal evolution of $\tau_{a_{\lambda}}$ estimation, we can define a residual signal as in classical approaches as follows :

$$
r(t)= \begin{cases}1 & \text { if } \dot{\tau}_{a_{\lambda}}=0 \text { and } \tau_{a_{\lambda}} \neq 0 \\ 0 & \text { otherwise }\end{cases}
$$


This signal is zero when there is no fault and it is equal to 1 when a fault occurs.

The above algorithm can be applied for all types of faults modelled by structured signals.

The case of sensor faults can be treated identically to the case of actuator faults. However, in contrast of the case of actuator faults, a sensor fault $f_{s_{j}}$ detection is accomplished from $y_{j}$ only. This makes easier the isolation of the faulty sensor.

In the following, without loss of generality, we focus our study on single input single output systems modeled by differential equations of order $n$ given in distributional framework by

$$
a_{n} y^{(n)}+\cdots+a_{0} y=\phi_{0}+b u
$$

where $\phi_{0}$ contains contribution of initial conditions.

The approach presented in this section is developed under the assumption that the exact order of the system is known and that no noise corrupt the signals. Before reviewing the questions of robustness, with respect to the model order and measurement noises, the proposed approach is extended in the next section in order to take into account some a priori information.

\section{ROBUSTNESS WITH RESPECT TO SYSTEM ORDER}

One of the most important parameters useful to apply the algorithm developed in section (III) is the order of the system. This section is devoted to the study of the algorithm behavior in the case of system over-modeling or system under-modeling of the considered system. Note that many methods are available in system identification framework to determine the system order [8].

Let $N$ be the real order of the system under consideration and $n$ the order of the associate model. Under the assumption of the occurrence of an actuator fault $f_{a}$ and a sensor fault $f_{s}$, the faulty system $\left(\sum\right)$ and model $(M)$ can be represented as

$$
\begin{aligned}
\left(\sum\right): \alpha_{N} y^{(N)} & +\cdots+\alpha_{0} y=\beta u+\psi_{0} \\
& +\beta f_{a}+\alpha_{N} f_{s}^{(N)}+\ldots+\alpha_{0} f_{s} \\
(M): a_{n} y^{(n)} & +\cdots+a_{0} y=b u+\phi_{0}+\mathfrak{E} \\
& +b f_{a}+a_{n} f_{s}^{(n)}+\ldots+a_{0} f_{s}
\end{aligned}
$$

Initial conditions are included in $\psi_{0}$ and $\phi_{0}$ which are distributions [12] with common support $\{0\}$ and order, respectively, $N-1$ and $n-1$.

$$
\begin{aligned}
\psi_{0} & =\sum_{k=1}^{N} \alpha_{k} \sum_{i=0}^{k-1} y^{(i)}(0) \delta^{(k-i-1)} \\
\phi_{0} & =\sum_{k=1}^{n} a_{k} \sum_{i=0}^{k-1} y^{(i)}(0) \delta^{(k-i-1)}
\end{aligned}
$$

$\mathfrak{E}$ contains modeling error such that (29) and (30) are consistent.

\section{A. Over-modeling}

In the case of an exact modeling ( $n=N$ and $\mathfrak{E}=0)$, we obtain an efficient algorithm to diagnose actuator and sensor faults. The same performance can be expected when the model order $n$ is greater than system order $N$ (overmodeling). Indeed, in this case, it is easy to see that

- the modeling error $\mathfrak{E}$ is zero,

- the annihilator of $\phi_{0}, f_{a}$ and $f_{s}^{(n)}$ (in (30)) cancels also the terms $\psi_{0}, f_{a}$ and $f_{s}^{(N)}$ (in (29)),

- estimates of $a_{N}, \ldots, a_{0}$ and $b$ given by the approach are exactly the estimates of the system parameters $\alpha_{N}, \ldots, \alpha_{0}$ and $\beta$ respectively.

Thus the steps of the proposed approach led to a problem of type (18). In order to illustrate this, let us consider a first order input-output system. A second order model is used to detect and identify an actuator fault modeled by $f_{a}(t)=$ $H(t-1)$. The faulty system $\left(\sum\right)$ and model $(M)$ can be represented as

$$
\begin{array}{cc}
\left(\sum\right): & \alpha_{1} y^{(1)}+\alpha_{0} y=\beta u+\alpha_{1} y(0) \delta+\beta H\left(t-\tau_{a}\right) \\
(M): & a_{2} y^{(2)}+a_{1} y^{(1)}+a_{0} y=b u+a_{2} y(0) \dot{\delta}+ \\
& \left(a_{2} \dot{y}(0)+a_{1} y(0)\right) \delta+b H\left(t-\tau_{a}\right)
\end{array}
$$

The estimation of $\tau_{a}$ (fault occurrence time) and $l_{a}$ (magnitude), based on model (33), is represented on figure (3).

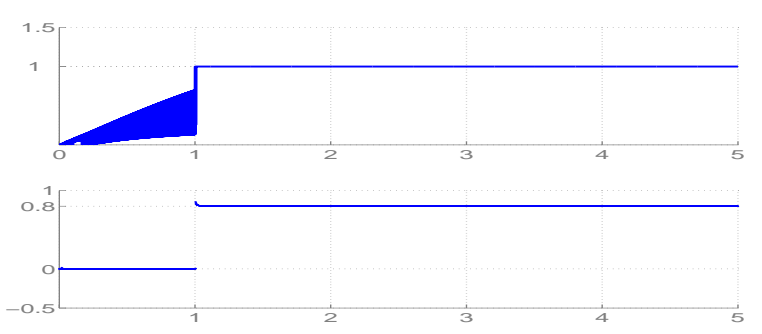

Fig. 3. estimation of $\tau_{a}$ (top) and $l_{a}$ (down)

\section{B. Under-modeling}

When the order of the system is under estimated, i.e $N>$ $n$, the associated equation (18) does not hold. Instead, we will have rather

$$
\left[A_{k}-\beta_{k-1} A_{k-1}-\ldots-\beta_{1} A_{1}\right] X=R \neq 0
$$

The $j^{\text {th }}$ element $(j=1, \ldots, m)$ of residual vector $R$ is given by

$\sum_{k=n+1}^{N} \alpha_{k} \underbrace{\int_{0}^{t} \ldots \int}_{p+j \text { times }} \Gamma\left(\left(-y+f_{s}\right)^{(k)}+\sum_{i=0}^{k-(n+1)} y^{(i)}(0) \delta^{(k-1-i)}\right) d \tau^{p+j}$

Indeed, annihilator $\Gamma$ of $\phi_{0}, f_{a}$ and $f_{s}^{(n)}$ (in (30)) satisfies

$\Gamma f_{s}^{(i)}=0, \forall i \leq n$ and $\Gamma \psi_{0}=\sum_{k=n+1}^{N} \sum_{i=0}^{k-(n+1)} y^{(i)}(0) \delta^{(k-1-i)}$ 
The residual vector is function of some initial conditions and derivatives of high order of $y$ and $f_{s}$ (in the case of sensor fault). When the difference of order is important, or when the parameters of high indices, appearing in (34) are not negligible compared to the parameters of low indices, then the proposed method may not identify the fault. These remarks are illustrated through figures (4) and (5). The first one is obtained with the system

$$
0.3 \ddot{y}+4.2 \dot{y}+9 y=9 u
$$

and the second one with

$$
4 \ddot{y}+4.2 \dot{y}+9 y=9 u
$$

These systems are characterized by the same static gain (equal to 1$)$ and poles $(-2.64 ;-11.35)$ and $(-0.52+1.4 i$; $-0.52-1.4 i)$ respectively. Both are corrupted by an actuator fault $f_{a}=-H(t-1.5)$. Using a first order model, we see on figure (4) that the estimates do not become stationary, but fluctuate around the true values, contrary to the case of overmodeling. Nevertheless, because of their low fluctuations, we can conclude to the occurrence of an actuator fault with magnitude (constant) $l_{a} \simeq-1$ at time $\tau_{a} \simeq 1.5$.

On the other side, as we can see on figure (5) none of the generalised eigenvalues obtained with $1^{\text {st }}$ order model admits a behavior close to the stationarity. These results are not useful to conclude to the occurrence or not of a fault.

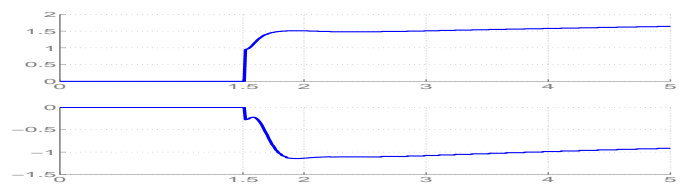

Fig. 4. Estimation of $\tau_{a}$ (top) and $l_{a}$ (down) obtained with model $0.3 \ddot{y}+$ $4.2 \dot{y}+9 y=9 u$

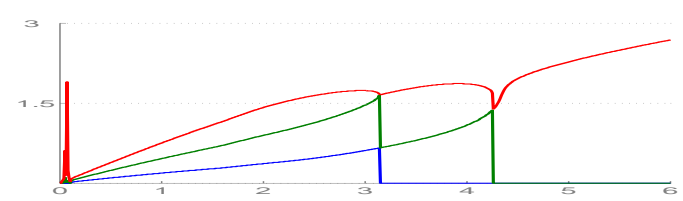

Fig. 5. Temporal evolution of all generalised eigenvalues obtained with model $4 \ddot{y}+4.2 \dot{y}+9 y=9 u$

Given the results of the study in this section, it is of interest to consider a higher order (but not too at risk of obtaining sparse matrices) to generate signals on which faults diagnosis is based.

\section{ROBUSTNESS WITH RESPECT TO MEASUREMENT NOISES}

The question of robustness of the proposed approach with respect to high frequency measurement noises is addressed in this section.

In addition to faults $f_{a}$ and $f_{s}$, measurement $y$ is assumed to be corrupted by an unstructured perturbation noted $\pi$. The generation of redundancy relations leads to
$\left[\left(A_{k}-\Delta A_{k}\right)-\beta_{k-1}\left(A_{k-1}-\Delta A_{k-1}\right)-\ldots-\beta_{1}\left(A_{1}-\Delta A_{1}\right)\right] X=0$

where matrices $A_{i}$ are expressed in terms of known signals $u$ and $y$, while matrices $\Delta A_{i}$ are linked to perturbation $\pi$.

The robustness analysis is based on

- the analysis of the filters generating elements of matrices $A_{i}$ and

- the properties of pseudospectra of matrix pencil $A_{2}-$ $\lambda A_{1}$ noted $\Lambda\left(A_{2}, A_{1}\right)$.

Studies of these two points are made in sections below.

\section{A. Analysis of the filters generating matrices $A_{i}$}

By considering annihilator $\Gamma$ from which we obtained equation (18) and based on properties

1) (Cauchy) $\int_{0}^{t} \ldots \int f(\tau) d \tau^{p}=\int_{0}^{t} \frac{(t-\tau)^{p-1}}{(p-1) !} f(\tau) d \tau$

2) $t^{k} \delta^{(n)}=\sum_{j=0}^{i n f(k, n)} C_{j}^{k}(-1)^{j} \frac{n !}{(n-j) !}\left[t^{k-j} y\right]^{(n-j)}$

3) (Newton) $(t-\tau)^{k}=\sum_{j=0}^{k}(-1)^{j} C_{j}^{k} t^{k-j} \tau^{j}$ matrices $A_{i}$ are reduced to the expression

$$
\begin{array}{r}
A_{i}(j, \mu)=\int_{0}^{t} f_{i, j, \mu}(t-\tau) y(\tau) d \tau, \mu=1, \ldots, n+1 \\
A_{i}(j, n+2)=\int_{0}^{t} g_{i, j}(t-\tau) u(\tau) d \tau
\end{array}
$$

where $f_{i, j, \mu}$ and $g_{i, j}$ are polynomial functions of appropriate degrees and depending on the assumptions of the diagnosis problem.

The transfer matrix between $e=\left[\begin{array}{l}u \\ y\end{array}\right]$ and $A_{i}(j,:)$, considered as linear filter, has impulse response

$$
h(t)=\left[\begin{array}{cc}
0 & f_{i, j, 1}(t) \\
\vdots & \vdots \\
0 & f_{i, j, n+1}(t) \\
g_{i, j}(t) & 0
\end{array}\right]
$$

This transfer matrix corresponds to a low-pass filter (according to the polynomial form of $f_{i, j, \mu}$ and $g_{i, j}$ ), i.e. only low frequencies pass and high frequencies (noise) are significantly attenuated. One can find the performance evaluation of this filter in discrete time domain in [6], where the authors approximate the integral using a trapezoidal discretization regularly spaced.

The choice of the annihilator $\Gamma$ is not unique. We obtain a filter of the same nature as previously by considering the differential operator given for $w \in \mathbb{R}^{+}$by

$$
\Gamma_{e x p}=e^{-w t} \Gamma
$$

The development of the proposed approach is not based on statistical-noise properties. When a priori knowledge of these properties is available, it can be taken into account to choose filter parameters $\left(f_{i, j, 1}, g_{i, j}, w, \cdots\right)$ in order to improve the robustness with respect to measurement noises. 


\section{B. $\epsilon$-pseudospectra of matrix pencils $A-\lambda B$}

The steps of our approach lead to a study of generalized eigenvalue of a couple of matrices $(A, B)$. In practice, the elements of these matrices are obtained by measurements, thus corrupted by error :

$$
A=\tilde{A}+\epsilon \Delta A, \quad B=\tilde{B}+\epsilon \Delta B
$$

where matrices $A$ and $B$ are expressed in terms of input signal $u$ and output signal $y$, while matrices $\Delta A$ and $\Delta B$ are linked to perturbation (or noise). In such situations, quantitative information obtained from only the spectra analysis of the matrices couple $(A, B)$ may be false. Also, note that traditional methods of solving generalized eigenvalues problem do not often give a solution. The robustness analysis can also be based on the properties of $\epsilon$-pseudospectra of matrix pencils $A-\lambda B$ ([2] and [13]).

For two matrices $A$ and $B$ in $\mathbb{R}^{m \times n}, \lambda$ is said to be a $\epsilon$ pseudo eigenvalue of the matrices couple $(A, B)$, if it exists a vector $\nu \neq 0$ (the associating pseudo eigenvector) such that

$$
\|(A-\lambda B) \nu\| \leq \epsilon
$$

The set of $\epsilon$-eigenvalues of $(A, B)$ is called $\epsilon$-pseudospectra of $(A, B)$ and it is noted $\Lambda_{\epsilon}(A, B)$. When the norm in (38) is the Euclidean norm, then

$$
\Lambda_{\epsilon}(A, B)=\left\{\lambda \in \mathbb{R}: \sigma_{\min }(A-\lambda B) \leq \epsilon\right\}
$$

where $\sigma_{\min }(M)$ means the smallest singular value of matrix $M$.

Let consider again the example of a bias diagnosis on a $1^{\text {st }}$ order system. The input and output signals are represented on figures (6) and (7). A centered gaussian white noise with variance 0.01 is added to the output $y$ before generating the two matrices $A_{2}$ and $A_{1}$. The input $u$ is also corrupted by the noise since the system is simulated in closed-loop using a $P I$ controller. Figure (8) shows the graphical results of the estimations (of $\tau_{a}$ and $l_{a}$ ) given by the proposed algorithms.

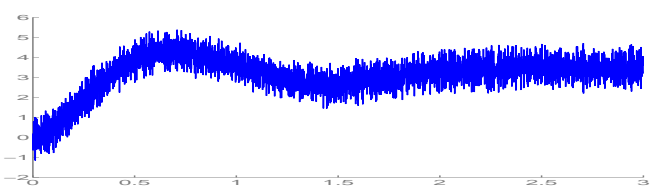

Fig. 6. Input signal $u$

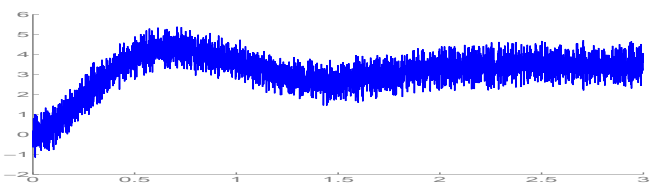

Fig. 7. Output signal $y$

Analysis of these figures confirms the robustness of the proposed approach regarding to additive noise with rapid fluctuations. One can find in [4], [9] and [10] other theoretical reasons explaining the robustness to noise.

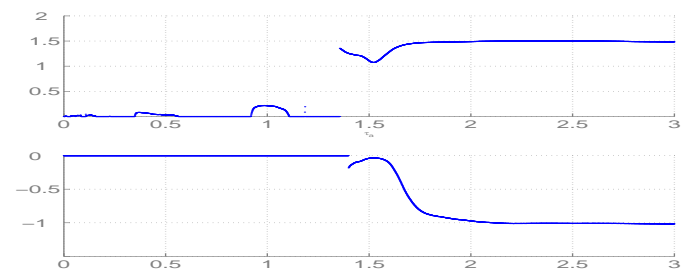

Fig. 8. Temporal evolution of $\tau_{a}$ (top) and $l_{a}$ (down) estimations obtained with $\Gamma_{e x p}=\left[e^{-0.3 t} t^{3} \frac{d}{d t}\right]-\tau_{a}\left[e^{-0.3 t} t^{2} \frac{d}{d t}\right]$

\section{CONCLUSION}

This paper has dealt with an algebraic approach to fault diagnosis as part of a new deterministic theory of estimation, based the functional calculus. We focus our study on the diagnosis of actuator and sensor faults in a class of uncertain linear continuous dynamic systems. Algorithms for detection, isolation and identification of faults are based on structural properties of the system and fault signals. The main advantage of this approach is that the system parameters can be unknown and we do not need to estimate them explicitly. Simulation results show that the proposed approach gives good results for fault diagnosis of uncertain linear systems. Because of the cancelation of the contribution of initial conditions and quick computations (due to explicit expressions), a local diagnosis can be made possible. This would also allow to extend the approach to systems slowly evolving over time. The analysis of robustness respect to the structure of faults will be future work.

\section{REFERENCES}

[1] L. Belkoura, "Identifiability and algebraic identification of time delay systems," in 9th IFAC Workshop on Time Delay System, Prague, Tchèque, 2010.

[2] G. Boutry, M. Elad, G. Golub, and P. Milanfar, "The generalized eigenvalue problem for non-square pencils using a minimal perturbation approach," SIAM Journal on Matrix Analysis and Applications, vol. 27, pp. 582-601, 2005.

[3] S. X. Ding, Model-Based Fault Diagnosis Techniques - Design Schemes Algorithms and tools. Springer, 2008.

[4] M. Fliess, "Analyse non standard du bruit," Comptes-Rendus de l'Académie des Sciences, Série 1, Mathématiques, vol. 342, pp. 797802, 2006.

[5] M. Fliess and H. Sira-Ramirez, "An algebraic framework for linear identification," ESAIM Control, Optimization and Calculus of Variations, vol. 9, pp. 151-168, 2003.

[6] F. A. García Collado, B. D’Andréa-Novel, M. Fliess, and H. Mounier, "Analyse fréquentielle des dérivateurs algébriques," in XXIIe Colloque GRETSI, Dijon France, 2009.

[7] R. Isermann, Fault-Diagnosis System. Berlin: Springer, 2006.

[8] L. Ljung, System identification (2nd ed.): theory for the user. Upper Saddle River, NJ, USA: Prentice Hall PTR, 1999.

[9] M. Mboup, "Parameter estimation for signals described by differential equations," Applicable Analysis : An International Journal, vol. 88, pp. 29-52, 2009.

[10] M. Mboup, C. Join, and M. Fliess, "Numerical differentiation with annihilators in noisy environnement," Numerical Algorithm, vol. 50, pp. 439-467, 2009.

[11] A. Moussa Ali, C. Join, and F. Hamelin, "Fault diagnosis of uncertain linear system using structural knowledge." in 7th IFAC Symposium on Fault Detection (Safeprocess), 2009.

[12] L. Schwartz, Théorie des distributions, 2nd ed. Paris: Hermann, 1966.

[13] T. G. Wright and L. N. Trefethen, "Pseudospectra of rectangular matrices," IMA J. of Numer. Anal., vol. 22, pp. 501-519, 2002. 